\title{
Existence of Best Proximity Point with an Application to Nonlinear Integral Equations
}

\author{
Shagun Sharma and Sumit Chandok \\ School of Mathematics, Thapar Institute of Engineering and Technology, Patiala 147004, India \\ Correspondence should be addressed to Sumit Chandok; sumit.chandok@thapar.edu
}

Received 10 July 2021; Accepted 17 August 2021; Published 8 October 2021

Academic Editor: Huseyin Isik

Copyright (C) 2021 Shagun Sharma and Sumit Chandok. This is an open access article distributed under the Creative Commons Attribution License, which permits unrestricted use, distribution, and reproduction in any medium, provided the original work is properly cited.

Using the idea of modified $\varrho$-proximal admissible mappings, we derive some new best proximity point results for $\varrho-\vartheta$-contraction mappings in metric spaces. We also provide some illustrations to back up our work. As a result of our findings, several fixed-point results for such mappings are also found. We obtain the existence of a solution for nonlinear integral equations as an application.

\section{Introduction and Preliminaries}

Problems originating in several disciplines of mathematical analysis, such as obtaining the existence of a solution for integral and differential equations, are solved using fixedpoint theory. The study of fixed points for nonself-mapping, on the contrary, is also fascinating. A nonself-contraction $\mathfrak{\Im}: \zeta \longrightarrow E$ does not necessarily have a fixed point for two given nonempty closed subsets $\zeta$ and $E$ of a complete metric space $\mathcal{\aleph}$. In this case, it is important to identify a point $\dot{u} \in \zeta$ such that $\wp\left(\dot{u}^{\prime}, \mathfrak{J} \dot{u}\right)$ is minimum. Essentially, if

$$
\wp\left(\dot{u}^{\prime}, \Im \dot{u}^{\prime}\right)=\operatorname{dist}(\zeta, E)=\inf \left\{\wp\left(\dot{u}^{\prime}, \widetilde{v}\right): \dot{u}^{\prime} \in \zeta, \widetilde{v} \in E\right\}
$$

where $\wp\left(\dot{u}^{\prime}, \mathfrak{J} \mathfrak{u}^{\prime}\right)$ is the minimum value $\operatorname{dist}(\zeta, E)$ and hence $\dot{u}$ is an approximate solution of equation $\Im u^{\prime}=\dot{u}$ with least possible error, such a solution is known as the best proximity point of mapping $\mathfrak{\Im}$.

Various academics have discovered a number of best proximity point theorems and associated fixed-point results in metric or normed linear spaces (see [1-7] and references cited therein). Inspired by the work of Geraghty [8] and Kutbi and Sintunavarat [9], we introduced a new class of nonself-contractive mappings known as $\varrho-\vartheta$-contraction. The goal of this work is to use the modified $\varrho$-proximal admissible mappings concept to obtain some best proximity point outcomes for $\varrho-\vartheta$-contraction mappings in metric space. On a metric space enriched with an arbitrary binary relation, some optimal proximity point results are proved. For such a class of mappings, we also obtain certain fixedpoint findings. As an application of our obtained results, we find the solution of a nonlinear integral equation.

The set of natural numbers and the set of real numbers are denoted by $\mathbb{N}$ and $\mathbb{R}$, respectively, throughout this work.

Let $\zeta$ and $E$ be two nonempty subsets of $(\aleph, \wp)$, a metric space. Define

$$
\begin{aligned}
\wp(\zeta, E)= & \inf \left\{\wp\left(\dot{u}^{\prime}, \widetilde{v}\right): \dot{u}^{\prime} \in, \widetilde{v} \in E\right\}, \\
\zeta_{0}= & \left\{\dot{u}^{\prime} \in \zeta: \text { there exists some } \widetilde{v}\right. \\
& \left.\cdot \in E \text { such that } \wp\left(\dot{u}^{\prime}, \widetilde{v}\right)=\wp(\zeta, £)\right\}, \\
E_{0}= & \{\widetilde{v} \in E: \text { there exists some } \dot{u} \\
& \left.\cdot \in \zeta \text { such that } \wp\left(\dot{u}^{\prime}, \widetilde{v}\right)=\wp(\zeta, £)\right\} .
\end{aligned}
$$

Definition 1. Let $(\zeta, E)$ be a pair of nonempty subsets of a metric space $(\aleph, \wp)$ with $\zeta_{0} \neq \varnothing$. Then, the pair $(\zeta, E)$ is said to have $£$-property (see [10]) if and only if, for any $\dot{u}_{1}, \dot{u}_{2} \in \zeta$ and $\widetilde{v}_{1}, \widetilde{v}_{2} \in E$, 


$$
\begin{aligned}
& \wp\left(\dot{u}_{1}, \widetilde{v}_{1}\right)=\wp(\zeta, E), \\
& \wp\left(\dot{u}_{2}, \widetilde{v}_{2}\right)=\wp(\zeta, E), \quad \text { then } \wp\left(\dot{u}_{1}^{\prime}, \dot{u}_{2}\right)=\wp\left(\widetilde{v}_{1}, \widetilde{v}_{2}\right) .
\end{aligned}
$$

Many authors have refined the concept of $\varrho$-admissible mappings developed by Samet [10] for proximal mappings (see $[11,12])$.

Definition 2. Let $\zeta$ and $E$ be two nonempty subsets of a metric space $(\aleph, \wp)$. A mapping $\mathfrak{\Im}: \zeta \longrightarrow E$ is called modified $\varrho$-proximal admissible if there exists a mapping $\varrho: E \times E \longrightarrow[0, \infty)$ such that $\varrho\left(\Im u_{0}^{\prime}, \Im u_{1}^{\prime}\right) \geq 1$ :

$$
\begin{aligned}
& \wp\left(\dot{x}_{1}, \Im \dot{u}_{0}\right)=\wp(\zeta, E), \\
& \wp\left(\dot{x}_{2}, \Im \dot{u}_{1}^{\prime}\right)=\wp(\zeta, E), \quad \text { then } \varrho\left(\Im x_{1}^{\prime}, \Im \dot{x}_{2}\right) \geq 1,
\end{aligned}
$$

for all $\dot{u}_{0}, \dot{u}_{1}, \dot{x}_{1}, \dot{x}_{2} \in \zeta$.

Let $(\aleph, \wp)$ be a metric space and ${ }^{\circledR}$ be a binary relation over $\aleph$. Denote

$$
\kappa={ }^{\circledR}+{ }^{\circledR}{ }^{-1}
$$

is the symmetric, transitive relation attached to ${ }^{\circledR}$. Clearly,

$$
\begin{array}{r}
\dot{u}, \widetilde{v} \in \aleph, \\
\dot{u} \kappa \widetilde{v} \Leftrightarrow \dot{u}^{\circledR} \widetilde{v} \text { or } \widetilde{v}^{\circledR} \dot{u}^{\prime} .
\end{array}
$$

Definition 3. Let $\zeta$ and $E$ be two nonempty subsets of metric space $(\aleph, \wp)$. A mapping $\mathfrak{\Im}: \zeta \longrightarrow E$ is called modified proximal comparative mapping; if $\mathfrak{J} \dot{u}_{0} \kappa \mathfrak{J} \dot{u}_{1}$,

$$
\begin{aligned}
& \wp\left(\dot{x}_{1}, \Im u_{0}^{\prime}\right)=\wp(\zeta, E), \\
& \wp\left(\dot{x}_{2}, \Im u_{1}^{\prime}\right)=\wp(\zeta, E), \quad \text { then } \Im^{\prime} x_{1} \kappa \Im x_{2}^{\prime},
\end{aligned}
$$

for all $\dot{u}_{0}, \dot{u}_{1}, \dot{x}_{1}, \dot{x}_{2} \in \zeta$.

Definition 4. Let $\aleph$ be a metric space. A mapping $\varrho$ : $\aleph \times$ $\aleph \longrightarrow[0, \infty)$ is called transitive [13] if it satisfies the following condition:

$$
\varrho\left(\dot{x}^{\prime}, \dot{y}^{\prime}\right) \geq 1, \varrho\left(\dot{y}^{\prime}, z^{\prime}\right) \geq 1 \text { implies } \varrho\left(\dot{x}^{\prime}, \dot{z}^{\prime}\right) \geq 1,
$$

for all $\dot{x}, \dot{y}, z^{\prime} \in \aleph$.

\section{Main Results}

To start with, we have the following definition:

Definition 5. Let $\zeta$ and $E$ be two nonempty subsets of a metric space $(\aleph, \wp)$. A mapping $\mathfrak{\Im}: \zeta \longrightarrow E$ is an $\varrho-\vartheta$ contraction mapping if there exist two functions $\varrho: E \times E \longrightarrow[0, \infty)$ and $\vartheta: \aleph \longrightarrow[0,1)$ for which $\vartheta\left(\Im\left(\dot{u}^{\prime}\right)\right) \leq \vartheta\left(\dot{u}^{\prime}\right)$ and limsup $\vartheta\left(\dot{u}^{\prime}\right)<1$ for all $\dot{u}^{\prime} \in \aleph$ such that

$$
\varrho\left(\Im\left(\dot{u}_{1}\right), \mathfrak{\Im}\left(\dot{u}_{2}\right)\right) \wp\left(\Im\left(\dot{u}_{1}\right), \mathfrak{\Im}\left(\dot{u}_{2}\right)\right) \leq \vartheta\left(\Im \dot{u}_{1}\right) \wp\left(\dot{u}_{1}, \dot{u}_{2}\right),
$$

for all $\dot{u}_{1}, \dot{u}_{2} \in \zeta$

Theorem 1. Let $\zeta$ and $E$ be two nonempty closed subsets of a complete metric space $\aleph$ such that $\zeta_{0}$ is nonempty. Let $\mathrm{Q}: E \times$ $E \longrightarrow[0, \infty)$ be transitive and $\mathfrak{\Im}: \zeta \longrightarrow E$ be continuous mapping satisfying the following assertions:

(i) $\mathfrak{\Im}$ is $\varrho-\vartheta$ contraction

(ii) $\mathfrak{I}\left(\zeta_{0}\right) \subseteq E_{0}$ and $(\zeta, E)$ satisfy the $£$-property

(iii) $\mathfrak{\Im}$ is modified $\varrho$-proximal admissible

Furthermore, suppose that there exists $\dot{u}_{0}, \dot{u}_{1} \in \zeta_{0}$ and $\mathfrak{\Im} \dot{u}_{0} \in E_{0}$ such that $\wp\left(\dot{u}_{1}^{\prime}, \mathfrak{\Im} \dot{u}_{0}\right)=\wp(\zeta, E)$ and $\varrho\left(\mathfrak{I} \dot{u}_{0}\right.$, $\left.\mathfrak{\Im} \dot{u}_{1}{ }^{0}\right) \geq 1$. Then, the mapping $\mathfrak{I}$ has a best proximity point.

Proof. By assumption, there exists $\dot{u}_{0}, \dot{u}_{1} \in \zeta_{0} \subseteq \zeta$ and $\Im \dot{u}_{0}^{\prime} \in \Im\left(\zeta_{0}\right) \subseteq E_{0} \subseteq E$ such that $\wp\left(\dot{u}_{1}^{\prime}, \Im \dot{u}_{0}^{\prime}\right)=\wp(\zeta, E)$ and $\varrho\left(\Im u_{0}^{\prime}, \Im u_{1}^{\prime}\right) \geq 1$.

Since $\dot{u}_{1}^{1} \in \zeta_{0}$, then $\mathfrak{\Im} \dot{u}_{1} \in \mathfrak{\Im}\left(\zeta_{0}\right) \subseteq E_{0}$. By definition of $E_{0}$, there exists $\dot{u}_{2} \in \zeta_{0}$ such that $\wp\left(\dot{u}_{2}, \mathfrak{J} \dot{u}_{1}\right)=\wp(\zeta, E)$. Since $\Im$ is modified $\varrho$-proximal admissible, and $\varrho\left(\Im \dot{u}_{0}, \mathfrak{\Im} \dot{u}_{1}\right) \geq 1$, we obtain

$$
\begin{aligned}
& \wp\left(\dot{u}_{1}^{\prime}, \Im \dot{u}_{0}^{\prime}\right)=\wp(\zeta, E), \\
& \wp\left(\dot{u}_{2}^{\prime}, \Im \dot{u}_{1}^{\prime}\right)=\wp(\zeta, E), \quad \text { then } \varrho\left(\Im \dot{u}_{1}^{\prime}, \Im \dot{u}_{2}^{\prime}\right) \geq 1 .
\end{aligned}
$$

Again, $\dot{u}_{2} \in \zeta_{0}$; then, $\mathfrak{\Im} \dot{u}_{2} \in \mathfrak{J}\left(\zeta_{0}\right) \subseteq E_{0}$. By definition of $E_{0}$, there exists $\dot{u}_{3} \in \zeta_{0}$ such that $\wp\left(\dot{u}_{3}, \mathfrak{J} \dot{u}_{2}\right)=\wp(\zeta, E)$. Since $\mathfrak{I}$ is modified $\varrho$-proximal admissible, and $\varrho\left(\Im \dot{u}_{1}, \mathfrak{s} \dot{u}_{2}\right) \geq 1$, we have

$$
\begin{aligned}
& \wp\left(\dot{u}_{2}, \Im \dot{u}_{1}^{\prime}\right)=\wp(\zeta, E), \\
& \wp\left(\dot{u}_{3}, \Im \dot{u}_{2}\right)=\wp(\zeta, E), \quad \text { then } \varrho\left(\Im \dot{u}_{2}^{\prime}, \Im \dot{u}_{3}\right) \geq 1 .
\end{aligned}
$$

Continuing in this fashion, we can construct a sequence $\left\{\dot{u}_{n}\right\}$ such that

$$
\left\{\begin{array}{l}
\wp\left(\dot{u}_{n+1}^{\prime}, \Im \dot{u}_{n}^{\prime}\right)=\wp(\zeta, E), \\
\wp\left(\dot{u}_{n}^{\prime}, \Im \dot{u}_{n-1}\right)=\wp(\zeta, E), \\
\varrho\left(\Im \dot{u}_{n}^{\prime}, \Im \dot{u}_{n+1}^{\prime}\right) \geq 1 .
\end{array}\right.
$$

As a pair $(\zeta, E)$ satisfies £-property, we have

$$
\wp\left(\dot{u}_{n}, \dot{u}_{n+1}\right)=\wp\left(\Im \dot{u}_{n-1}, \Im \dot{u}_{n}\right)
$$


For $n \in \mathbb{N}$, we have

$$
\begin{aligned}
\wp\left(\dot{u}_{n}, \dot{u}_{n+1}\right)= & \wp\left(\Im\left(\dot{u}_{n-1}\right), \Im\left(\dot{u}_{n}\right)\right) \\
& \leq \varrho\left(\Im u_{n-1}, \Im u_{n}^{\prime}\right) \wp\left(\Im u_{n-1}^{\prime}, \Im u_{n}\right) \\
& \leq \vartheta\left(\Im \dot{u}_{n-1}\right) \wp\left(\dot{u}_{n-1}^{\prime}, \dot{u}_{n}\right) \\
& \leq \vartheta\left(\dot{u}_{n-1}^{\prime}\right) \wp\left(\dot{u}_{n-1}, \dot{u}_{n}\right) \\
& <\wp\left(\dot{u}_{n-1}^{\prime}, \dot{u}_{n}\right),
\end{aligned}
$$

for all $n \in \mathbb{N}$. Therefore, the sequence $\left\{\wp\left(\dot{u}_{n-1}, \dot{u}_{n}\right)\right\}$ is strictly decreasing and

$$
\lim _{n \longrightarrow+\infty} \wp\left(\dot{u}_{n-1}, \dot{u}_{n}\right)=\dot{d}
$$

for some $d^{\prime} \geq 0$.

Next, we claim that $\dot{d}=0$. Assume, on the contrary, that $d>0$. On taking limit as $n \longrightarrow+\infty$ in equation (14), we obtain that

$$
\begin{aligned}
\lim _{n \longrightarrow+\infty} \frac{\wp\left(\dot{u}_{n}, \dot{u}_{n+1}\right)}{\wp\left(\dot{u}_{n-1}, \dot{u}_{n}\right)} \leq \vartheta\left(\dot{u}_{n-1}\right), \\
1 \leq \vartheta\left(\dot{u}_{n-1}\right),
\end{aligned}
$$

which is a contradiction. Therefore, $\dot{d}=0$.

Next, we show that $\left\{\dot{u}_{n}\right\}$ is a Cauchy sequence. Using triangle inequality, (12) and (13), we have, for all $m, n \in \mathbb{N}$ and $m>n$,

$$
\begin{aligned}
& \wp\left(\dot{u}_{m}, \dot{u}_{n}\right) \leq \wp\left(\dot{u}_{m}, \dot{u}_{m+1}\right)+\wp\left(\dot{u}_{m+1}, \dot{u}_{n+1}\right)+\wp\left(\dot{u}_{n+1}, \dot{u}_{n}\right) \\
& =\wp\left(\dot{u}_{m}, \dot{u}_{m+1}\right)+\wp\left(\mathfrak{I} \dot{u}_{m}, \mathfrak{J} \dot{u}_{n}\right)+\wp\left(\dot{u}_{n+1}, \dot{u}_{n}\right)
\end{aligned}
$$

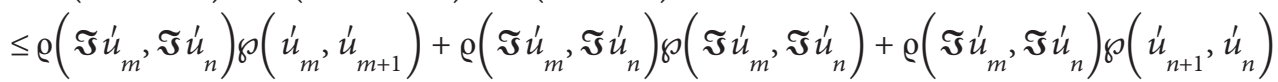

$$
\begin{aligned}
& \leq \varrho\left(\mathfrak{J} \dot{u}_{m}, \mathfrak{J} \dot{u}_{n}\right)\left[\wp\left(\dot{u}_{m}, \dot{u}_{m+1}\right)+\wp\left(\dot{u}_{n+1}, \dot{u}_{n}\right)\right]+\vartheta\left(\Im \dot{u}_{m}\right) \wp\left(\dot{u}_{m}, \dot{u}_{n}\right) \\
& \leq \varrho\left(\Im \dot{u}_{m}, \mathfrak{s} \dot{u}_{n}\right)\left[\wp\left(\dot{u}_{m}, u_{m+1}\right)+\wp\left(\dot{u}_{n+1}, u_{n}\right)\right]+\vartheta\left(\dot{u}_{m}\right) \wp\left(\dot{u}_{m}, u_{n}\right) \text {. }
\end{aligned}
$$

It implies that

$$
\begin{aligned}
& \left(1-\vartheta\left(\dot{u}_{m}\right)\right) \wp\left(\dot{u}_{m}, \dot{u}_{n}\right) \\
& \quad \leq \varrho\left(\Im \dot{u}_{m}, \Im \dot{u}_{n}\right)\left[\wp\left(\dot{u}_{m}, \dot{u}_{m+1}\right)+\wp\left(\dot{u}_{n+1}, \dot{u}_{n}\right)\right] .
\end{aligned}
$$

On taking limit as $m, n \longrightarrow+\infty$ in (18), we obtain that

$$
\lim _{m, n \longrightarrow+\infty} \wp\left(\dot{u}_{m}, \dot{u}_{n}\right)=0 \text {. }
$$

Therefore, $\left\{\hat{u}_{n}\right\}$ is a Cauchy sequence in $\zeta$. Since $\zeta$ is a closed subset of a complete metric $\aleph$, we obtain

$$
\lim _{n \rightarrow+\infty} \dot{u}_{n}=\dot{u}^{\prime}
$$

for some $\dot{u} \in \zeta$. Since $\mathfrak{I}$ is continuous,

$$
\lim _{n \longrightarrow+\infty} \mathfrak{I}\left(\dot{u}_{n}\right)=\mathfrak{J}\left(\dot{u}^{\prime}\right)
$$

for some $\mathfrak{I}\left(\imath^{\prime}\right) \in E$. By (12), we obtain

$$
\wp\left(\dot{u}^{\prime}, \mathfrak{\Im} \dot{u}^{\prime}\right)=\lim _{n \longrightarrow+\infty} \wp\left(\dot{u}_{n+1}, \mathfrak{\Im}\left(\dot{u}_{n}\right)\right)=\wp(\zeta, E) .
$$

Hence, $\dot{u}$ is a best proximity point of $\mathfrak{I}$.

The following hypotheses can be used to substitute $\mathfrak{\Im}$ 's continuity hypothesis in Theorem 1 .
$(\mathfrak{R})$ If $\left\{\dot{u}_{n}\right\}$ is a sequence in $\aleph$ such that $\varrho\left(\Im \dot{u}_{n}, \Im \dot{u}_{n+1}^{\prime}\right)$ $\geq 1$, for all $n$ and $\dot{u}_{n} \longrightarrow \dot{u} \in \mathcal{N}$ as $n \longrightarrow \infty$, then there exists a subsequence $\left\{\dot{u}_{n(k)}^{\prime}\right\}$ of $\left\{\dot{u}_{n}\right\}$ such that $\varrho\left(\Im \dot{u}_{n(k)}^{\prime}, \Im \dot{u}^{\prime}\right) \geq 1$, for all $k$.

Theorem 2. Let $\zeta$ and $E$ be two nonempty closed subsets of a complete metric space $\aleph$ such that $\zeta_{0}$ is nonempty. Let $\mathrm{Q}: E \times$ $E \longrightarrow[0, \infty)$ be transitive and $\mathfrak{\Im}: \zeta \longrightarrow E$ be a mapping satisfying the following assertions:

(i) $\mathfrak{\Im}$ is $\varrho-\vartheta$ contraction

(ii) $\mathfrak{\Im}\left(\zeta_{0}\right) \subseteq E_{0}$ and $(\zeta, E)$ satisft the $£$-property

(iii) $\mathfrak{\Im}$ is modified $\varrho$-proximal admissible

Furthermore, suppose that ( $R$ ) holds and there exists $\dot{u}_{0}, \dot{u}_{1}$ $\in \zeta_{0}$ and $\mathfrak{\Im} u_{0}^{\prime} \in E_{0}$ such that $\wp\left(u_{1}^{\prime}, \mathfrak{\Im} u_{0}\right)=\wp(\zeta, E)$ and $\varrho\left(\Im u_{0}^{\prime}\right.$, $\left.\mathfrak{J} u_{1}^{\prime}\right) \geq 1$. Then, the mapping $\mathfrak{I}$ has a best proximity point.

Proof. Following the proof of Theorem 1, the sequence $\left\{u_{n}\right\}$ is Cauchy and converges to some $\dot{u}^{*}$ in $(\aleph, \wp)$.

From our assumption and using (12), there exists a subsequence $\left\{\dot{u}_{n(k)}\right\}$ of $\left\{\dot{u}_{n}\right\}$ such that $\varrho\left(\Im \dot{u}_{n(k)}, \mathfrak{J} \dot{u}^{*}\right) \geq 1$, for all $k$.

Now, we shall show that $\mathfrak{\Im}$ has a best proximity point. Using triangle inequality and (12), we obtain 


$$
\begin{aligned}
& \wp\left(\dot{u}_{n(k)+1}, \mathfrak{\Im} \dot{u}^{*}\right) \leq \wp\left(\dot{u}_{n(k)+1}, \mathfrak{\Im} \dot{u}_{n(k)}\right)+\wp\left(\Im \dot{u}_{n(k)}, \mathfrak{\Im} \dot{u}^{\prime *}\right) \\
& \leq \wp(\zeta, E)+\varrho\left(\Im \dot{u}_{n(k)}, \Im \dot{u}^{*}\right) \wp\left(\Im \dot{u}_{n(k)}, \Im \dot{u}^{\prime *}\right) \\
& \leq \wp(\zeta, E)+\vartheta\left(\Im \dot{u}_{n(k)}\right) \wp\left(\dot{u}_{n(k)}, \dot{u}^{*}\right) \\
& \leq \wp(\zeta, E)+\vartheta\left(\dot{u}_{n(k)}\right) \wp\left(\dot{u}_{n(k)}, \dot{u}^{*}\right) \text {. }
\end{aligned}
$$

Again, by triangle inequality and equation (23), we have

$$
\begin{aligned}
\wp(\zeta, E) & \leq \wp\left(\dot{u}^{*}, \Im \dot{u}^{*}\right) \leq \wp\left(\dot{u}^{*}, \dot{u}_{n(k)+1}\right)+\wp\left(\dot{u}_{n(k)+1}, \mathfrak{\Im} \dot{u}^{*}\right) \\
& \leq \wp\left(\dot{u}^{*}, \dot{u}_{n(k)+1}\right)+\wp(\zeta, E)+\vartheta\left(\dot{u}_{n(k)}\right) \wp\left(\dot{u}_{n(k)}, \dot{u}^{*}\right) .
\end{aligned}
$$

Taking $k \longrightarrow+\infty$ in (24), we have

$$
\wp(\zeta, E)=\wp\left(\iota^{*}, \Im \iota^{*}\right) \text {. }
$$

This shows that $\mathfrak{I}$ has a best proximity point.

\section{Best Proximity Points on a Metric Space Endowed with an Arbitrary Binary Relation}

Using modified proximal comparative mapping, we establish some optimal proximity point findings on a metric space equipped with an arbitrary binary relation in this section.

Theorem 3. Let $\zeta$ and $E$ be two nonempty closed subsets of a complete metric space $(\aleph, \wp)$ such that $\zeta_{0}$ is nonempty and ${ }^{\circledR}$ be a binary relation over $\aleph$. Suppose that $\mathfrak{\Im}: \zeta \longrightarrow E$ is a continuous mapping satisfying the following assertions:

(i) There exists $\vartheta: \aleph \longrightarrow[0,1)$ such that, for all $\dot{u}_{1}, \dot{u}_{2} \in \zeta, \dot{u}_{1} \kappa \dot{u}_{2}$,

$$
\wp\left(\Im \dot{u}_{1}^{\prime}, \Im \dot{u}_{2}^{\prime}\right) \leq \vartheta\left(\dot{u}_{1}\right)\left(\wp\left(\dot{u}_{1}^{\prime}, \dot{u}_{2}\right)\right) \text {. }
$$

(ii) $\mathfrak{\Im}\left(\zeta_{0}\right) \subseteq E_{0}$ and $(\zeta, E)$ satisfy the $£$-property.

(iii) $\mathfrak{I}$ is modified proximal comparative mapping.

If there exists $\dot{u}_{3}, \dot{u}_{4} \in \zeta_{0}$ and $\mathfrak{\Im} \dot{u}_{3} \in E_{0}$ such that $\wp\left(\dot{u}_{4}, \mathfrak{J} \dot{u}_{3}\right)=\wp(\zeta, E), \dot{u}_{3} \kappa \dot{u}_{4}^{\prime}$, then the mapping $\mathfrak{I}$ has a best proximity point.

Proof. Define a mapping $\varrho: E \times E \longrightarrow \mathbb{R}$ by

$$
\varrho\left(\Im \dot{u}_{1}^{\prime}, \Im \dot{u}_{2}^{\prime}\right)= \begin{cases}2, & \text { if } \mathfrak{\Im} \dot{u}_{1} \kappa \mathfrak{s} \dot{u}_{2}, \\ 1, & \text { otherwise. }\end{cases}
$$

Suppose that $\varrho\left(\Im \dot{u}_{3}, \Im \dot{u}_{4}^{\prime}\right) \geq 1$, such that

$$
\left\{\begin{array}{l}
\wp\left(\dot{u}_{4}, \Im u_{3}^{\prime}\right)=\wp(\zeta, E), \\
\wp\left(\dot{u}_{5}, \Im \mathfrak{u}_{4}^{\prime}\right)=\wp(\zeta, E) .
\end{array}\right.
$$
obtain

Hold for some $\dot{u}_{3}, \dot{u}_{4}, \dot{u}_{5} \in \zeta$. By definition of $\varrho$, we

$$
\begin{array}{r}
\mathfrak{J}_{3}^{\prime} \kappa \Im \dot{u}_{4}^{\prime}, \\
\wp\left(\dot{u}_{4}, \Im \dot{u}_{3}\right)=\wp(\zeta, E), \\
\wp\left(\dot{u}_{5}, \Im \dot{u}_{4}\right)=\wp(\zeta, E) .
\end{array}
$$

Condition (iii) implies that $\mathfrak{\Im} \dot{u}_{4} \kappa \mathfrak{\Im} \dot{u}_{5}$, which gives us from the definition of $\varrho$ that $\varrho\left(\Im \dot{u}_{4},{ }^{4}, \mathfrak{J} \dot{u}_{5}\right) \geq 1$. This shows $\mathfrak{I}$ is a modified $\varrho$-proximal admissible mapping.

Furthermore, using assumption, we have

$$
\begin{aligned}
& \wp\left(\dot{u}_{4}, \Im \dot{u}_{3}\right)=\wp(\zeta, E), \\
& \varrho\left(\Im \dot{u}_{3}^{\prime}, \Im \dot{u}_{4}^{\prime}\right) \geq 1 .
\end{aligned}
$$

Condition (i) implies that

$\varrho\left(\Im \dot{u}_{1}, \Im \dot{u}_{2}\right) \wp\left(\Im \dot{u}_{1}, \Im \dot{u}_{2}\right) \leq \vartheta\left(\dot{u}_{1}\right)\left(\wp\left(\dot{u}_{1}, \dot{u}_{2}\right)\right), \quad \forall \dot{u}_{1}, \dot{u}_{2} \in \zeta$.

Thus, all the conditions of Theorem 1 are satisfied; then, $\mathfrak{I}$ has a best proximity point.

We may replace the continuity hypothesis of $\mathfrak{J}$ in Theorem 3 by the following hypothesis.

$\left(\Re^{*}\right)$ If $\left\{u_{n}^{\prime}\right\}$ is a sequence in $\aleph$ such that $\Im u_{n}^{\prime} \kappa \Im u_{n+1}^{\prime}$ for all $n$ and $u_{n} \longrightarrow u^{\prime} \in \mathcal{N}$ as $n \longrightarrow+\infty$, then there exists a subsequence $\left\{u_{n(k)}^{\prime}\right\}$ of $\left\{u_{n}^{\prime}\right\}$ such that $\Im u_{n(k)}^{\prime} \kappa \Im u^{\prime}$, for all $k$.

Theorem 4. Let $\zeta$ and $E$ be two nonempty closed subsets of a complete metric space $(\aleph, \wp)$ such that $\zeta_{0}$ is nonempty and ${ }^{\circledR}$ be a binary relation over $\aleph$. Suppose that $\mathfrak{\Im}: \zeta \longrightarrow E$ is a mapping satisfying the following assertions:

(i) There exists $\vartheta: \aleph \longrightarrow[0,1)$ such that, for all $u_{1}^{\prime}, \dot{u}_{2} \in \zeta, \dot{u}_{1} \kappa \dot{u}_{2}$,

$$
\wp\left(\Im \dot{u}_{1}^{\prime}, \Im \dot{u}_{2}^{\prime}\right) \leq \vartheta\left(\dot{u}_{1}\right)\left(\wp\left(\dot{u}_{1}, \dot{u}_{2}\right)\right)
$$

(ii) $\mathfrak{\Im}\left(\zeta_{0}\right) \subseteq E_{0}$ and $(\zeta, E)$ satisfy the $£$-property.

(iii) $\mathfrak{I}$ is modified proximal comparative mapping.

Furthermore, we assume that $\left(\mathfrak{R}^{*}\right)$ holds and there exists $\dot{u}_{3}, \dot{u}_{4} \in \zeta_{0}$ and $\mathfrak{\Im} \dot{u}_{3} \in E_{0}$ such that $\wp\left(\dot{u}_{4}, \mathfrak{\Im} \dot{u}_{3}\right)=\mathfrak{\Im}(\zeta, E)$, $\dot{u}_{3} \kappa \dot{u}_{4}$. Then, the mapping $\mathfrak{I}$ has a best proximity point.

Proof. By evaluating the mapping $\varrho$ defined in Theorem 3 and noting that condition $\left(\mathfrak{R}^{*}\right)$ implies condition $(\mathfrak{R})$, the result is derived from Theorem 2 .

\section{Consequences and Related Results}

In this section, we obtain some results on the existence of fixed points as a result of our findings.

If we take $\zeta=E=\aleph$ in Theorem 1, we obtain the following result: 
Corollary 1 (see Theorem 10 in [9]). Let $\aleph$ be a complete metric space. Let $\mathrm{\varrho}: \aleph \times \aleph \longrightarrow[0, \infty)$ be transitive and $\mathfrak{\Im}: \aleph \longrightarrow \aleph$ be continuous mapping satisfying the following assertions:

(i) $\mathfrak{I}$ is $\varrho-\vartheta$ contraction

(ii) $\mathfrak{I}$ is o admissible

(iii) There exists $\dot{u}_{0} \in \zeta$ such that $\varrho\left(\dot{u}_{0}, \mathfrak{\Im} \dot{u}_{0}\right) \geq 1$

Then, the mapping $\mathfrak{I}$ has a fixed point.

By considering $\varrho\left(\dot{u}_{1}, \dot{u}_{2}\right)=1$, for all $\dot{u}_{1}, \dot{u}_{2} \in \zeta$ and $\vartheta\left(\dot{u}_{1}\right)=k ; k \in[0,1)$, for all $\dot{u}_{1} \in \aleph$ in Corollary 1 , we get the famous Banach contraction theorem.

Corollary 2 (see [14]). Let $\aleph$ be a complete metric space and $\mathfrak{\Im}: \aleph \longrightarrow \mathcal{N}$ be a mapping satisfying the following assertion:

$$
\wp\left(\Im \dot{u}_{1}^{\prime}, \mathfrak{s} \dot{u}_{2}\right) \leq k \wp\left(\dot{u}_{1}, \dot{u}_{2}\right)
$$

for all $\dot{u}_{1}, \dot{u}_{2} \in \aleph$. Then, the mapping $\mathfrak{I}$ has a fixed point.

If we take $\zeta=E=\aleph$ in Theorem 2, we obtain the following result.

Corollary 3 (see Theorem 12 in [9]). Let $\aleph$ be a complete metric space. Let $\varrho: \aleph \times \aleph \longrightarrow[0, \infty)$ be transitive and $\mathfrak{\Im}: \aleph \longrightarrow \aleph$ be a mapping satisfying the following assertions:

(i) $\mathfrak{\Im}$ is $\varrho-\vartheta$ contraction

(ii) $\mathfrak{\Im}$ is @ admissible

(iii) There exists $\dot{u}_{0} \in \aleph$ such that $\varrho\left(\dot{u}_{0}, \mathfrak{\Im} \dot{u}_{0}\right) \geq 1$

(iv) (R) holds

Then, the mapping $\mathfrak{I}$ has a fixed point.

If we take $\zeta=E=\aleph$ in Theorems 3 and 4 , we obtain the following fixed-point results:

Corollary 4. Suppose that $\mathfrak{\Im}: \aleph \longrightarrow \aleph$ is a continuous mapping on a metric space $(\aleph, \wp)$ with a binary relation ${ }^{\circledR}$ over $\aleph$, satisfying

$$
\wp\left(\Im \dot{u}_{1}, \Im \dot{u}_{2}^{\prime}\right) \leq \vartheta\left(\dot{u}_{1}\right)\left(\wp\left(\dot{u}_{1}, \dot{u}_{2}\right)\right)
$$

for $u_{1}^{\prime}, u_{2}^{\prime} \in \aleph, u_{1}^{\prime} \kappa u_{2}^{\prime}$, where $\vartheta: \aleph \longrightarrow[0,1)$. If there exists $u_{*}^{\prime} \in$ $\aleph$ a such that $\dot{u}_{*}^{\prime} \kappa \mathfrak{J}^{\prime}$, then the mapping $\mathfrak{\Im}$ has a fixed point.

Corollary 5. Suppose that $\mathfrak{\Im}: \aleph \longrightarrow \aleph$ is a self-mapping on a metric space $(\aleph, \wp)$ with a binary relation ${ }^{\circledR}$ over $\aleph$, satisfying

$$
\wp\left(\Im \dot{u}_{1}^{\prime}, \Im \dot{u}_{2}^{\prime}\right) \leq \vartheta\left(\dot{u}_{1}^{\prime}\right)\left(\wp\left(\dot{u}_{1}^{\prime}, \dot{u}_{2}\right)\right)
$$

for $\dot{u}_{1}, \dot{u}_{2} \in \aleph, \dot{u}_{1} \kappa \dot{u}_{2}$, where $\vartheta: \aleph \longrightarrow[0,1)$. If there exists $\dot{u}_{*} \in \aleph$ such that $\dot{u}_{*} \kappa \mathfrak{I} \dot{u}_{*}$ and $\left(\mathfrak{R}^{*}\right)$ holds, then the mapping $\mathfrak{\Im}$ has a fixed point.

\section{Examples}

We give several illustrations that support our findings in this section.
Example 1. Consider $\aleph=\mathbb{R}^{2}$ with metric

$$
\wp\left(\widetilde{v}, \dot{u}_{*}\right)=\sqrt{\left(\widetilde{v}_{1}-\widetilde{v}_{2}\right)^{2}+\left(\dot{u}_{1}^{*}-\dot{u}_{2}^{*}\right)^{2}},
$$

for all $\widetilde{v}=\left(\widetilde{v}_{1}, \dot{u}_{1}^{*}\right), \dot{u}^{*}=\left(\widetilde{v}_{2}, u_{2}^{*}\right) \in \mathbb{R}^{2}$.

Suppose $\quad \zeta=\left\{\left((1 / 2), \dot{u}_{1}\right): 0 \leq \dot{u}_{1} \leq 1\right\} \quad$ and $E=\left\{\left(0, \dot{u}_{1}\right): 0 \leq \dot{u}_{1} \leq 1\right\}$, such that $\wp(\zeta, E)=(1 / 2)$.

Define $\mathfrak{\Im}: \zeta \longrightarrow E$ by

$$
\mathfrak{J}\left(\dot{u}_{1}^{\prime}\right)= \begin{cases}(0,1), & \dot{u}_{1}=\left(\frac{1}{2}, 1\right), \\ \left(0, \frac{a}{4}\right): 0 \leq \mathfrak{a}^{\prime} \leq \dot{u}_{1}, & \text { otherwise, }\end{cases}
$$

for all $\dot{u}_{1} \in \zeta$.

Define $\varrho: E \times E \longrightarrow[0, \infty)$ by

$$
\varrho\left(\left(0, \dot{u}_{1}^{*}\right),\left(0, \dot{u}_{2}^{*}\right)\right)= \begin{cases}1, & \text { if } \dot{u}_{1}^{*}, \dot{u}_{2}^{*} \in\left[0, \frac{1}{4}\right], \\ 2, & \text { otherwise, }\end{cases}
$$

then $\varrho$ is transitive. If $z_{1}^{\prime}=\left((1 / 2), \dot{u}_{1}\right)$ and $z_{2}^{\prime}=\left((1 / 2), \dot{u}_{2}\right)$ in $\zeta$, for $\dot{u}_{1}, \dot{u}_{2} \in[0,(1 / 2)]$, then

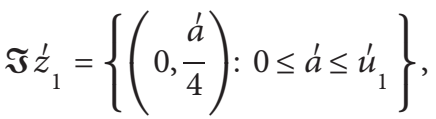

$$
\begin{aligned}
& \mathfrak{\Im} \stackrel{\prime}{z}_{2}=\left\{\left(0, \frac{a}{4}\right): 0 \leq a^{\prime} \leq \dot{u}_{2}\right\} \text {. }
\end{aligned}
$$

Also, $\varrho\left(\Im \dot{z}_{1}, \Im \dot{z}_{2}\right)=1$ and $\wp\left(\dot{u}_{1}, \Im \frac{\prime}{z_{1}}\right)=(1 / 2)=$ $\wp(\zeta, E)$ and $\wp\left(\dot{u}_{2}, \mathfrak{\Im} \dot{z}_{2}^{\prime}\right)=(1 / 2)=\wp(\zeta, E)$ if and only if $\dot{u}_{1}, \dot{u}_{2} \in\{((1 / 2),(\dot{x} / 4)) ; 0 \leq \dot{x} \leq(1 / 2)\} . \quad$ Then, $\varrho\left(\Im \dot{u}_{1}^{\prime}, \mathfrak{J} \dot{u}_{2}\right)=1$. This shows $\Im$ is a modified $\varrho$-proximal admissible and $\mathfrak{\Im}$ is continuous. Since $\zeta_{0}=\zeta$ and $E_{0}=E$, then $\mathfrak{I}\left(\zeta_{0}\right) \subseteq E_{0}$, for each $\dot{u}_{1} \in \zeta_{0}$.

Next, we prove, that $\mathfrak{J}$ is $\varrho-\vartheta$ contraction. Let $\vartheta(t)=(1 / 3)$, for all $t \in \aleph$. Take $z_{1}^{\prime}=\left((1 / 2), \dot{u}_{1}\right)$ and $z_{2}=$ $\left((1 / 2), \dot{u}_{2}\right)$ in $\zeta$, where $0 \leq \dot{u}_{1}, \dot{u}_{2} \leq(1 / 2)$. Consider

$$
\begin{aligned}
\wp\left(\Im z_{1}^{\prime}, \mathfrak{J} z_{2}^{\prime}\right) & =\sqrt{(0-0)^{2}+\left(\frac{\dot{u}_{1}}{4}-\frac{\dot{u}_{2}}{4}\right)^{2}} \\
& =\sqrt{\left(\frac{\dot{u}_{1}}{4}-\frac{\dot{u}_{2}}{4}\right)^{2}}=\left(\frac{\dot{u}_{1}}{4}-\frac{\dot{u}_{2}}{4}\right) \\
& =\frac{1}{4}\left(\dot{u}_{1}-\dot{u}_{2}\right) \leq \frac{1}{3}\left(\dot{u}_{1}-\dot{u}_{2}\right) .
\end{aligned}
$$




$$
\varrho\left(\Im z_{1}^{\prime}, \Im z_{2}^{\prime}\right) \wp\left(\Im z_{1}^{\prime}, \Im z_{2}^{\prime}\right) \leq \vartheta\left(z_{1}^{\prime}\right) \wp\left(z_{1}^{\prime}, z_{2}\right),
$$

for all $z_{1}, \dot{z}_{2} \in \zeta$. Hence, $\mathfrak{I}$ is a $\varrho-\vartheta$ contraction. All conditions of Theorem 1 are satisfied and $\mathfrak{I}$ has a best proximity point $((1 / 2), 1)$.

Example 2. Consider $\aleph=\mathbb{R}^{2}$ with metric

$$
\wp\left(\widetilde{v}, \dot{u}_{*}\right)=\sqrt{\left(\widetilde{v}_{1}-\widetilde{v}_{2}\right)^{2}+\left(\dot{u}_{1}^{*}-u_{2}^{*}\right)^{2}}
$$

for all $\widetilde{v}=\left(\widetilde{v}_{1}, \dot{u}_{1}^{*}\right), \mathfrak{u}^{*}=\left(\widetilde{v}_{2}, u_{2}^{*}\right) \in \mathbb{R}^{2}$. Suppose that

$$
\begin{aligned}
& \zeta=\left\{\left(\dot{u}_{1}, \dot{u}_{2}\right): \dot{u}_{1}^{2}+\dot{u}_{2}^{2}=3 \text { and } \dot{u}_{2} \geq 0\right\}, \\
& E=\left\{\left(\dot{u}_{1}, \dot{u}_{2}\right): \dot{u}_{1}^{2}+\dot{u}_{2}^{2}=1 \text { and } \dot{u}_{2} \geq 0\right\} .
\end{aligned}
$$

Then, $\wp(\zeta, E)=2$. Define $\mathfrak{I}: \zeta \longrightarrow E$ by

$$
\mathfrak{J}\left(\dot{u}_{1}, \dot{u}_{2}\right)=\frac{\left(\dot{u}_{1}, \dot{u}_{2}\right)}{3},
$$

for all $\left(\dot{u}_{1}, \dot{u}_{2}\right) \in \zeta$.

Define $\varrho: \aleph \times \aleph \longrightarrow[0, \infty)$ by

$$
\varrho\left(\left(u_{1}^{\prime}, u_{1}^{*}\right),\left(u_{2}, u_{2}^{*}\right)\right)=2,
$$

for all $\left(\dot{u}_{1}, \dot{u}_{1}^{*}\right),\left(\dot{u}_{2}, \dot{u}_{2}^{*}\right) \in \aleph$. Hence, $\varrho$ is transitive. If $\dot{z}_{1}, \dot{z}_{2} \in \zeta, \quad{ }^{1} \quad{ }^{1}$ then ${ }^{2} \varrho\left(\Im \dot{z}_{1}, \Im z_{2}^{\prime}\right)=2>1, \wp\left(\dot{u}_{1}, \Im \dot{z}_{1}\right)=$ $2=\wp(\zeta, E)$ and $\wp\left(\dot{u}, \mathfrak{\Im} \dot{z}_{2}\right)=2=\wp(\zeta, E)$ if and only if $\dot{u}_{1}, \dot{u}_{2} \in\{(0,3),(3,0)\}$. Therefore, $\varrho\left(\Im u_{1}, \Im \dot{u}_{2}\right)=2>1$. This shows $\mathfrak{I}$ is a modified $\varrho$-proximal admissible and $\mathfrak{I}$ is continuous. Since $\zeta_{0}=\zeta$ and $E_{0}=E$, then $\mathfrak{I}\left(\zeta_{0}\right) \subseteq E_{0}$, for each $\dot{u}_{1} \in \zeta_{0}$.

Next, we prove that $\mathfrak{I}$ is $\varrho-\vartheta$ contraction. in $\zeta$; then,

Let $\vartheta(t)=(1 / 2)$. Take $\dot{z}_{1}=\left(\dot{x}_{1}, \dot{y}_{1}\right)$ and $\dot{z}_{2}=\left(\dot{x}_{2}, \dot{y}_{2}\right)$

$$
\begin{aligned}
\wp\left(\mathfrak{J} \dot{z}_{1}, \mathfrak{J} \dot{z}_{2}\right) & =\sqrt{\left(\frac{\dot{x}_{1}}{3}-\frac{\dot{x}_{2}}{3}\right)^{2}+\left(\frac{\dot{y}_{1}}{3}-\frac{\dot{y}_{2}}{3}\right)^{2}} \\
& =\sqrt{\frac{1}{3^{2}}\left(\dot{x}_{1}-\dot{x}_{2}\right)^{2}+\frac{1}{3^{2}}\left(\dot{y}_{1}-\dot{y}_{2}\right)^{2}} \\
& =\frac{1}{3} \sqrt{\left(\dot{x}_{1}-\dot{x}_{2}\right)^{2}+\left(\dot{y}_{1}-\dot{y}_{2}\right)^{2}} \\
& =\frac{1}{3} \wp\left(\dot{z}_{1}, \dot{z}_{2}\right) .
\end{aligned}
$$

It implies that

$$
\varrho\left(\Im z_{1}^{\prime}, \Im z_{2}^{\prime}\right) \wp\left(\Im z_{1}^{\prime}, \Im\left(z_{2}^{\prime}\right)\right) \leq \vartheta\left(z_{1}^{\prime}\right) \wp\left(z_{1}, z_{2}\right) \text {, }
$$

for all $\dot{z}_{1}, \dot{z}_{2} \in \aleph$. Therefore, $\mathfrak{I}$ is a $\varrho-\vartheta$ contraction. All conditions of Theorem 1 are satisfied and $\mathfrak{I}$ has a best proximity point $(3,0)$.

\section{Application to Integral Equations}

In this section, we obtain the solution of integral equation as an application of our obtained results.

If we take $\zeta=E=\aleph$ in Theorem 1, we obtain the solution of nonlinear integral equation.

Theorem 5. Let $C\left[a^{\prime}, b^{\prime}\right]$ be the set of all continuous functions on closed interval $[\dot{a}, b]$, with metric defined by

$$
\wp\left(\dot{u}^{\prime}, v^{\prime}\right)=\sup _{\dot{t} \in[a, b]}\left|\dot{u}^{\prime}\left(\dot{t}^{\prime}\right)-\dot{v}^{\prime}\left(t^{\prime}\right)\right|,
$$

for all $\dot{u}^{\prime}, \dot{v} \in \mathcal{C}\left[\dot{a}, b^{\prime}\right]$. Consider the nonlinear integral equation:

$$
\dot{u}(\dot{t})=v(\dot{t})+\int_{a}^{\dot{b}} \omega(\dot{t}, \dot{x}, \dot{u}(\dot{x})) \mathrm{d} \dot{x},
$$

where $t^{\prime} \in\left[a, b^{\prime}\right], \quad v:\left[a^{\prime}, b^{\prime}\right] \longrightarrow \mathbb{R}$, and $\omega:\left[a^{\prime}, b^{\prime}\right] \times\left[a^{\prime}, b^{\prime}\right] \times$ $\dot{u}^{\prime}\left[a^{\prime}, b^{\prime}\right] \longrightarrow \mathbb{R}$ for each $\dot{u} \in \mathcal{C}\left[a^{\prime}, b^{\prime}\right]$. Suppose that the following statements hold:

(i) $v$ is continuous on $\left[\dot{a}^{\prime}, \dot{b}^{\prime}\right]$ and $\omega\left(t^{\prime}, \dot{x}, \dot{u}(\dot{x})\right)$ is integrable with respect to $\dot{x}$ on $[\dot{a}, \dot{b}]$

(ii) $\Im \dot{u} \in C\left[\mathfrak{a}^{\prime}, b^{\prime}\right], \quad$ for all $\dot{u} \in \mathcal{C}\left[\dot{a}^{\prime}, b^{\prime}\right]$, where $\Im \mathfrak{u}^{\prime}(\dot{t})=v(t)+\int_{\dot{a}}^{\dot{b}} \omega(\dot{t}, \dot{x}, \dot{u}(\dot{x})) d \dot{x}, \quad$ for all $\dot{t} \in[a, b]$

(iii) For all $\dot{u} \in C[\dot{a}, \dot{b}], \dot{u}(\dot{x}) \geq 0$, and $\Im \dot{u}(\dot{x}) \geq 0$, for all $\dot{x} \in\left[\dot{a}^{\prime}, b^{\prime}\right]$

(iv) For all $\dot{x}, \dot{t} \in\left[\dot{a}, b^{\prime}\right]$ and $\dot{u}, \dot{v} \in C[\dot{a}, \dot{b}]$, $\omega\left(t^{\prime}, \dot{x}, \dot{u}^{\prime}\left(\dot{x}^{\prime}\right)\right)$ $-\omega\left(t^{\prime}, \dot{x}, \dot{v}^{\prime}(\dot{x})\right) \mid \leq\left(k^{\prime} / b^{\prime}-a^{\prime}\right)\left(\max \left|\dot{u}(\dot{x})-\dot{v}^{\prime}(\dot{x})\right|\right) ;$ $k^{\prime} \in[0,1)$

(v) There exists $\dot{u}_{1} \in \mathcal{C}\left[\dot{a}^{\prime}, b^{\prime}\right]$ such that $\dot{u}_{1}\left(t^{\prime}\right) \geq 0$ and $\mathfrak{J}_{u_{1}}^{\prime}\left(t^{\prime}\right) \geq 0$, for all $t^{\prime} \in\left[{ }^{\prime}, b^{\prime}\right]$ $C[a, b]$

Then, nonlinear integral equation (49) has a solution in Proof. Define a mapping $\mathfrak{\Im}: \mathcal{C}\left[a^{\prime}, b^{\prime}\right] \longrightarrow \subset\left[a^{\prime}, b^{\prime}\right]$ by

$$
\Im \mathfrak{u}^{\prime}(\dot{t})=v(\dot{t})+\int_{\dot{a}}^{\dot{b}} \omega(\dot{t}, \dot{x}, \dot{u}(\dot{x})) \mathrm{d} \dot{x},
$$

for all $\dot{u} \in \mathcal{C}\left[\mathfrak{a}^{\prime}, b^{\prime}\right]$ and for all $t^{\prime} \in\left[\mathfrak{a}^{\prime}, b^{\prime}\right]$. Then, $\mathfrak{\Im}$ is a continuous mapping.

Define a mapping $\varrho: C\left[a^{\prime}, b^{\prime}\right] \times C\left[a^{\prime}, b^{\prime}\right] \longrightarrow \mathbb{R}$ by 


$$
\varrho\left(\dot{u}^{\prime}, \hat{v}^{\prime}\right)= \begin{cases}2, & \text { if } \dot{u}^{\prime}(\dot{x}), \dot{v}^{\prime}(\dot{x}) \in[0, \infty), \dot{x} \in\left[\dot{a}, b^{\prime}\right], \\ 1, & \text { otherwise. }\end{cases}
$$

We shall show that $\mathfrak{I}$ is a modified $\varrho$-proximal admissible mapping. Indeed, for $\dot{u}, \dot{v} \in \mathcal{C}[\hat{a}, b]$ such that $\varrho\left(\Im \dot{u}^{\prime}, \mathfrak{J} \dot{v}\right) \geq 1$, we have $\dot{u}(\dot{x}), \dot{v}(\dot{x}) \geq 0$, for all $\dot{x} \in\left[\dot{a}^{\prime} b^{\prime}\right]$. It follows from condition (iii) that $\mathfrak{\Im} \dot{u}(\dot{x}), \mathfrak{J} \dot{v}(\dot{x}) \geq 0$.

Therefore, $\varrho\left(\mathfrak{J} \dot{u}(\dot{x}), \mathfrak{I} \dot{v}^{\prime}(\dot{x})\right)>1$, and hence, $\mathfrak{I}$ is a modified $\varrho$-proximal admissible mapping and $\varrho$ is transitive. Now, we claim that $\mathfrak{I}$ is a $\varrho-\vartheta$ contraction. Let $\vartheta(\dot{x})=k^{\prime}$, for all $\dot{x} \in[a, b]$. Consider

$$
\begin{aligned}
& \left|\Im \dot{u}^{\prime}(\dot{x})-\Im \dot{J}^{\prime}(\dot{x})\right|=\left|\int_{\dot{a}}^{b} \omega(\dot{t}, \dot{x}, \dot{u}(\dot{x})) \mathrm{d} \dot{x}-\int_{\dot{a}}^{b} \omega(\dot{t}, \dot{x}, \dot{v}(\dot{x})) \mathrm{d} \dot{x}\right| \\
& \leq \int_{\dot{a}}^{\dot{b}}|\omega(\dot{t}, \dot{x}, \dot{u}(\dot{x}))-\omega(\dot{t}, \dot{x}, \dot{v}(\dot{x}))| \mathrm{d} \dot{x} \\
& \leq \frac{\dot{k}^{\prime}}{b^{\prime}-a^{\prime}}(\max |\dot{u}(\dot{x})-\dot{v}(\dot{x})|) \int_{\dot{a}}^{\dot{b}} \mathrm{~d}(\dot{x}) \\
& \leq \frac{\mathfrak{k}^{\prime}}{b^{\prime}-\mathfrak{a}^{\prime}}\left(\dot{b}^{\prime}-\mathfrak{a}^{\prime}\right)(\max |\dot{u}(\dot{x})-\dot{v}(\dot{x})|) \\
& \leq \dot{k}(\wp(\dot{u}, \dot{v})) \\
& \leq \mathfrak{V}(\dot{u})\left(\wp\left(\dot{u}, v^{\prime}\right)\right) .
\end{aligned}
$$

It implies that

$$
\varrho\left(\mathfrak{I}\left(\dot{u}^{\prime}\right), \mathfrak{I}\left(\hat{v}^{\prime}\right)\right)_{\wp}\left(\mathfrak{I}\left(\dot{u}^{\prime}\right), \mathfrak{I}\left(v^{\prime}\right)\right) \leq \mathfrak{\vartheta}\left(\dot{u}^{\prime}\right)\left(\wp\left(\dot{u}^{\prime}, \hat{v}^{\prime}\right)\right) .
$$

Then, $\Im$ is a $\varrho-\vartheta$ contraction. Let $\left\{\Im u_{n}^{\prime}\right\} \subseteq \complement\left[a^{\prime}, b^{\prime}\right]$ such that $\varrho\left(\Im \dot{u}_{n}, \Im \dot{u}_{n+1}^{\prime}\right) \geq 1$ and $\lim _{n \longrightarrow+\infty} \Im \dot{u}_{n}=\Im u^{\prime} \in \subset\left[\dot{a}^{\prime}, \dot{b}\right]$. Then, $\dot{u}(\dot{x}), \dot{u}_{n}(\dot{x}) \in[0, \infty)$, for all $\dot{x}^{\prime} \in\left[\dot{a}^{\prime}, b^{\prime}\right]$ and $n \geq 0$. Therefore, $\varrho\left(\Im u^{\prime}, \Im u^{\prime}\right)>1$, for all $n \geq 1$. Therefore, we conclude all the hypotheses of Theorem 1 are satisfied. Thus, equation (49) has a solution $\dot{u} \in \mathcal{C}\left[\mathfrak{a}^{\prime}, \mathfrak{b}^{\prime}\right]$.

\section{Conclusion}

Using the concept of modified $\varrho$-proximal admissible in the setting of metric space $(\aleph, \wp)$, we find some novel best proximity point results for $\varrho-\vartheta$-contraction mappings. Many known results in the literature are also generalized by our findings.

\section{Data Availability}

No data were used to support this study.

\section{Conflicts of Interest}

The authors declare that they have no conflicts of interest.

\section{Acknowledgments}

The first author is grateful for a Junior Research Fellowship from the UGC-CSIR. The second author is grateful for the research funding 02011/11/2020/NBHM (RP)/R\&D-II/7830 from the NBHM, DAE, India.

\section{References}

[1] S. Sadiq Basha and P. Veeramani, "Best proximity pair theorems for multifunctions with open fibres," Journal of Approximation Theory, vol. 103, no. 1, pp. 119-129, 2000.

[2] A. A. Eldred and P. Veeramani, "Existence and convergence of best proximity points," Journal of Mathematical Analysis and Applications, vol. 323, no. 2, pp. 1001-1006, 2006.

[3] K. Fan, "Extensions of two fixed point theorems of F. E. Browder," Mathematische Zeitschrift, vol. 112, no. 3, pp. 234-240, 1969.

[4] M. Gabeleh and H.-P. A. Künzi, "Equivalence of the existence of best proximity points and best proximity pairs for cyclic and noncyclic nonexpansive mappings," Demonstratio Mathematica, vol. 53, no. 1, pp. 38-43, 2020.

[5] W. Kirk and N. Shahzad, Fixed Point Theory in Distance Spaces, Springer, Cham, Switzerland, 2014.

[6] S. Reich, "Approximate selections, best approximations, fixed points, and invariant sets," Journal of Mathematical Analysis and Applications, vol. 62, no. 1, pp. 104-113, 1978.

[7] F. Vetro, "Fixed point for $\alpha-\Theta-\Phi$-contractions and first-order periodic differential problem," Revista de la Real Academia de Ciencias Exactas, Físicas y Naturales Serie A. Matemáticas, vol. 113, no. 3, pp. 1823-1837, 2019.

[8] M. A. Geraghty, "On contractive mappings," Proceedings of the American Mathematical Society, vol. 40, no. 2, p. 604, 1973.

[9] M. A. Kutbi and W. Sintunavarat, "Ulam-Hyers stability and well-posedness of fixed point problems for $\alpha-\lambda$ contraction mapping in metric spaces," Abstract and Applied Analysis, vol. 2014, Article ID 268230, 6 pages, 2014.

[10] B. Samet, "Some results on best proximity points," Journal of Optimization Theory and Applications, vol. 159, no. 1, pp. 281-291, 2013.

[11] M. U. Ali, T. Kamram, and N. Shahzad, "Best proximity point for $\alpha-\psi$-proximal contractive multimaps," Abstract and Applied Analysis, vol. 2014, p. 6, Article ID 181598, 2014.

[12] A. Hussain, T. Kanwal, and A. A. Rawashdeh, "Global best approximate solutions for set valued contraction in $b$-metric spaces with applications," Journal of Computational and Applied Mathematics, pp. 293-313, 2018.

[13] W. Sintunavarat, "Generalized Ulam-Hyers Stability, wellposedness, and limit shadowing of fixed point problems for $\alpha-\beta$ contraction mapping in metric spaces," The Scientific World Journal, vol. 2014, Article ID 569174, 7 pages, 2014.

[14] S. Banach, "Sur les opérations dans les ensembles abstraits et leur application aux équations intégrales," Fundamenta Mathematicae, vol. 3, pp. 133-181, 1922. 